\title{
CT/MRI LI-RADS version 2018 versus CEUS LI-RADS version 2017 in the diagnosis of primary hepatic nodules in patients with high- risk hepatocellular carcinoma
}

\author{
Kun $\mathrm{Lv}^{1,2 \#}$, Xin Cao ${ }^{1,3 \#}$, Yinlei Dong ${ }^{4}$, Daoying Geng ${ }^{1,3}$, Jun Zhang ${ }^{1,2}$ \\ ${ }^{1}$ Department of Radiology, Huashan Hospital, Fudan University, Shanghai, China; ${ }^{2}$ Institute of Functional and Molecular Medical Imaging, Fudan \\ University, Shanghai, China; ${ }^{3}$ Center for Shanghai Intelligent Imaging for Critical Brain Diseases Engineering and Technology Research, Shanghai, \\ China; ${ }^{4}$ Department of Pancreatic Surgery, Pancreatic Disease Institute, Huashan Hospital, Fudan University, Shanghai, China \\ Contributions: (I) Conception and design: D Geng; (II) Administrative support: J Zhang; (III) Provision of study materials or patients: K Lv; (IV) \\ Collection and assembly of data: K Lv, X Cao; (V) Data analysis and interpretation: J Zhang, Y Dong; (VI) Manuscript writing: All authors; (VII) \\ Final approval of manuscript: All authors. \\ "These authors contributed equally to this work. \\ Correspondence to: Daoying Geng; Jun Zhang. Department of Radiology, Huashan Hospital, Fudan University, 12 Wulumuqi Middle Road, Shanghai \\ 200040, China. Email: gdy_2019@163.com; zhj81828@163.com.
}

Background: This study aimed to explore the application value of computed tomography/magnetic resonance imaging (CT/MRI) liver imaging reporting and data system (LI-RADS) version 2018 and contrast-enhanced ultrasound (CEUS) LI-RADS version 2017 in high-risk hepatocellular carcinoma (HCC) patients and to conduct a comparative analysis.

Methods: This study enrolled 250 high-risk HCC patients with 259 primary hepatic nodules from June 2017 to June 2020. Two investigators used a single-blind method to classify all nodules. The $u$-test, $t$-test, and Kappa test were performed. The sensitivity, specificity, positive predictive value (PPV), negative predictive value and receiver operating characteristic curves of LR-5 and LR-M in the diagnosis of HCC and non-HCC malignancy were respectively calculated.

Results: CT/MRI LI-RADS v2018 and CEUS LI-RADS v2017 showed substantial agreement interobservers, and there was a moderate agreement inter-modality. The specificity and PPV of HCC and nonHCC malignancies in CT/MRI LR-5/M were higher than CEUS. The areas under the curve (AUC) of CT/ MRI LR-5 and LR-M were 0.794 and 0.777, and the AUC of CEUS LR-5 and LR-M were 0.720 and 0.718 , respectively.

Conclusions: Two modalities have substantial agreement inter-observers and moderate agreement intermodalities. The diagnostic accuracy of HCC of CT/MRI LR-5 and non-HCC malignancy of CT/MRI LR-M are higher than CEUS.

Keywords: Hepatocellular carcinoma (HCC); computed tomography; magnetic resonance imaging; ultrasonography

Submitted Mar 04, 2021. Accepted for publication Apr 16, 2021.

doi: 10.21037/atm-21-1035

View this article at: http://dx.doi.org/10.21037/atm-21-1035

\section{Introduction}

Primary liver cancer is the second leading cause of cancerrelated death worldwide. Hepatocellular carcinoma (HCC) is the most common primary liver cancer, accounting for approximately $90 \%$ of cases (1). Currently, HCC is reportedly the fifth most common cancer in the world and the third leading cause of cancer-related mortality (2). The incidence rate varies by geographic 
region. The annual incidence rate in East Asia and SubSaharan Africa is higher, exceeding 15 per 100,000. However, in Europe and the United States, the incidence of HCC has also been rising rapidly and will continue to rise in the next 10 years (3-5). Additionally, more than $80 \%$ of patients diagnosed with HCC reportedly have cirrhosis. The incidence of HCC in non-cirrhotic patients is $0.1-0.8$ per 100 people per year, and in patients with cirrhosis is 2.2-4.3 per 100 people per year (2). Therefore, cirrhosis or hepatitis B should be considered as a risk factor for HCC, and diagnosis of hepatic nodules with high-risk factors for HCC is very important.

Imaging is indispensable in the diagnosis of HCC. Unlike most cancers, HCC in patients with cirrhosis or hepatitis B can be diagnosed non-invasively and highly accurately based on multi-phase computed tomography/magnetic resonance imaging (CT/MRI) imaging features (6). However, some lesions, including HCC, non-HCC malignant tumors, and non-malignant lesions cannot be accurately diagnosed by ultrasound (US) and CT/MRI due to the complexity and similarity of their imaging characteristics. The American College of Radiology (ACR) has issued a diagnostic program, namely the liver imaging reporting and data system (LI-RADS), including US LI-RADS for address screening/surveillance, CT/MRI and contrast-enhanced ultrasound (CEUS) LI-RADS for diagnosis, and treatment response LI-RADS for response to liver-directed therapy, to describe the characteristics of focal liver lesions in highrisk HCC patients, and provide standardization for HCC screening, monitoring, diagnosis, and treatment response assessment. The CT/MRI LI-RADS have been improved several times based on previous versions, and the latest version has been released, namely CT/MRI LI-RADS v2018. To our knowledge, the comparative analysis between CT/MRI LI-RADS v2018 and CEUS LI-RADS v2017 in high-risk HCC patients with primary hepatic nodules has not been reported. Therefore, this study aimed to explore the application value of the two classification modalities in primary hepatic nodules and to conduct a comparative analysis.

We present the following article in accordance with the STARD reporting checklist (available at http://dx.doi. org/10.21037/atm-21-1035).

\section{Methods}

The study was conducted in accordance with the Declaration of Helsinki (as revised in 2013). The study was approved by institutional ethics committee of Huashan Hospital, Fudan University (No.: KY2021-066) and individual consent for this retrospective analysis was waived.

\section{Study participants}

This study consecutively and retrospectively collected samples from 967 patients who underwent CEUS and CE-CT/MRI examination of the liver from June 2017 to June 2020. Inclusion criteria: (I) liver cirrhosis or chronic hepatitis B virus infection; (II) primary hepatic nodules; (III) with pathological result. Exclusion criteria: (I) $<18$ years old, (II) liver cirrhosis due to congenital liver fibrosis or vascular disease, such as hereditary hemorrhagic telangiectasia, Budd-Chiari syndrome, chronic portal vein occlusion, congestive heart disease or diffuse nodular hyperplasia of the liver, (III) liver cancer undergone treatment, (IV) extrahepatic tumor or a definite history of metastasis, and $(\mathrm{V})$ more than 3 hepatic nodules were excluded to better evaluate the application value of CT/MRI/CEUS LI-RADS in the primary hepatic nodules. The interval between the two modalities was no more than 1 week, and the pathological tissue samples were obtained by surgery or biopsy within 2 weeks after the completion of CT/MRI and CEUS.

\section{Imaging acquisition}

\section{CEUS}

CEUS was conducted on a Mindray Resona-7 Scanner (Mindray Medical Solutions; Shenzhen, China). The frequency of the convex transducer was $1-5 \mathrm{MHz}$, and the mechanical index for CEUS was 0.08. The patients were placed in supine position and left decubitus position, and the images and videos were obtained by the attending physician who had worked for more than 10 years. The contrast media $(2.4 \mathrm{~mL})$, SonoVue (SonoVue; Bracco SpA, Milan, Italy), was administered through the antecubital vein followed by a flush with $5 \mathrm{~mL} 0.9 \%$ saline. After the contrast media injection was completed, the images were acquired according to the arterial phase (usually occurs from about $10-20$ to $30-45 \mathrm{~s}$ after contrast injection), portal venous phase (lasts from about $30-45 \mathrm{~s}$ to $2 \mathrm{~min}$ after contrast injection), late phase (lasts from end of portal venous phase until there is unequivocal clearance of microbubbles from the circulation at about 4-6 min). The B-mode images and contrast images were dual-screen displayed and obtained side by side. The observation time 
of each nodule was about 4-6 min. When there are two nodules, it is carried out in two times. The second time is performed after the previous contrast has been unequivocal clearance from the circulation. The time interval is about 10 minutes. The patients did not report any adverse effects.

\section{CT/MRI}

The CT scan was performed using a multi-slice spiral CT scanner (Somatom Definition AS; Siemens Healthineers, Erlangen, Germany). Contrast-enhanced scan was performed after plain scan. The contrast media $(80 \mathrm{~mL})$, iohexol (350 mgL/mL, Beilu Pharmaceutical Co., Ltd.), was injected through the antecubital vein at a rate of $3.0-3.5 \mathrm{~mL} / \mathrm{s}$. The arterial, venous, and delayed phases were performed after injection of the contrast media at 25-30, 55-60, and $180 \mathrm{~s}$, respectively. The scanning parameters included, tube voltage, $120 \mathrm{kv}$, tube current, $143 \mathrm{mAs}$, matrix, $512 \times 512$, field of view, $350 \mathrm{~mm}$, and thickness, $3 \mathrm{~mm}$. The MRI scan was performed using a 3.0-T MRI scanner (Discovery 750, GE Healthcare, Milwaukee, WI) equipped with 16-channel body coils. After the completion of T1-weighted imaging, T2-weighted imaging, diffusionweighted imaging, contrast-enhanced scan was performed. First, $10 \mathrm{~mL}(0.1 \mathrm{mmol} / \mathrm{kg})$ of the contrast media, gadolinium-diethylenetriamine penta-acetic acid (GdDTPA, Hokuriku Seiyaku Co., Ltd., Katsuyama, Japan), was injected intravenously at a rate of $2.0 \mathrm{~mL} / \mathrm{s}$. Next, the solution was flushed with $10 \mathrm{~mL} 0.9 \%$ sodium chloride. The arterial, portal venous and delayed phases were performed after injection of the contrast media at 15-20, $50-60$, and $180 \mathrm{~s}$, respectively. The scanning parameters included repetition time, $5.7 \mathrm{~ms}$; echo time, $1.9 \mathrm{~ms}$; field of view, $360 \mathrm{~mm}$; matrix 1 , voxel $1.4 \times 1.9$; and thickness, $5 \mathrm{~mm}$.

\section{Image analysis}

The study retrospectively analyzed the CEUS images, videos, and CT/MRI images obtained through the above methods (for simultaneous CT and MRI scans, MRI images were used). Pathological results as a reference standard. Two observers have 6 (XC) and 15 (JZ) years of radiology work experience respectively, and independently performed CT/ MRI and CEUS classification of all nodules in turn, and did not disclose any clinical information with the exception of information on patients with cirrhosis or chronic hepatitis B. When the classification was inconsistent, images were re-evaluated together until a consensus was reached. The two observers carefully studied the classification process of ACR CEUS LI-RADS v2017 and ACR CT/MRI LI-RADS v2018 (https://www.acr.org) before the nodule classification. LI-RADS includes LR-1 (definitely benign) to LR-5 (definitely HCC), LR-M, and LR-TIV (Tumor in vein). LR-M does not specifically refer to HCC, but it suggests that there may be intrahepatic cholangiocellular carcinoma (ICC), combined hepatocellular cholangiocarcinoma, or other rarer non-HCC malignancies.

\section{Statistics analysis}

Quantitative variables are expressed as mean \pm standard deviation. The SPSS software (SPSS Inc., Chicago, Illinois, USA, 1999) was used to perform $t$-test on the nodule sizes measured by the two modalities, and the Cohen's Kappa test (7) was used to analyze the agreement between the two classification modalities and between observers. The degrees of agreement were considered as no agreement at Kappa value $\leq 0$; none to slight at $0.01-0.20$; fair, $0.21-0.40$; moderate, 0.41-0.60; substantial, 0.61-0.80; and almost perfect agreement, $0.81-1$. Taking pathological diagnosis as a reference, the sensitivity, specificity, positive predictive value (PPV), and negative predictive value of LR-5 and LR-M in the diagnosis of HCC and non-HCC malignancy were respectively calculated for the two classification modalities, and then the $u$-test was used to determine whether there were statistical differences. Finally, the MedCalc software (version 15.2.2; www.medcalc.org) was used to analyze the diagnostic performance of the two classification modalities for the diagnosis of malignant liver lesions through receiver operating characteristic (ROC) curves. The test was considered significant at $\mathrm{P}<0.05$.

\section{Results}

\section{Clinical characteristics}

A total of 250 patients with 259 nodules were enrolled in this study (Figure 1), including 186 males and 64 females. The age range was $24-87$ years, and the average age was $61.32 \pm 10.76$ years. Nodule size measured by CT/MRI was $7-190 \mathrm{~mm}$, with an average of $51.46 \pm 35.06 \mathrm{~mm}$, and that measured by CEUS was approximately $6-158 \mathrm{~mm}$, with an average of $52.39 \pm 33.52 \mathrm{~mm}$. There was no statistical difference in nodule size measured by the two modalities based on $t$-test $(\mathrm{P}>0.05$, Table 1$)$. 


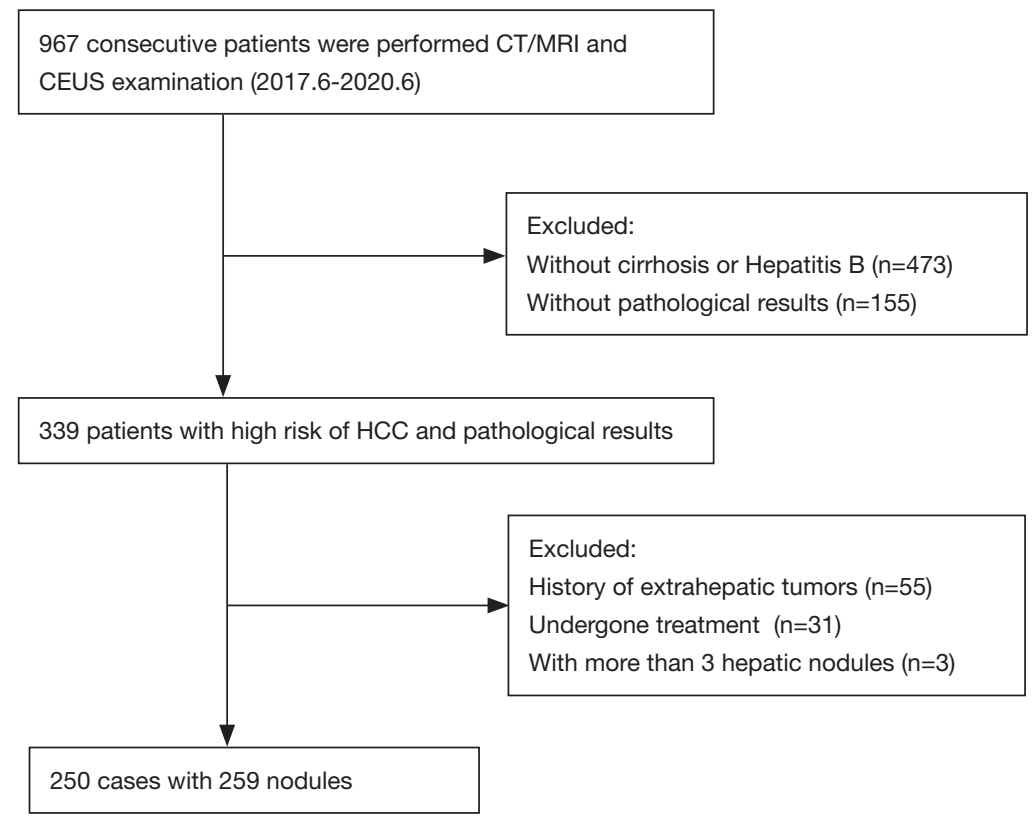

Figure 1 Flow chart of patients' enrollment. HCC, hepatocellular carcinoma; CEUS, contrast enhanced ultrasound.

Table 1 Patient characteristics $(\mathrm{n}=250)$

\begin{tabular}{lc}
\hline Characteristic & Value \\
\hline Age (years) (range/mean \pm SD) & $24-87(61.32 \pm 10.76)$ \\
Gender $(\mathrm{M} / \mathrm{F})$ & $186 / 64$ \\
Lesion size $(\mathrm{mm})(\text { range/mean } \pm \mathrm{SD})^{\star}$ & \\
CT/MRI & $7-190(51.46 \pm 35.06)$ \\
CEUS & $6-158(52.39 \pm 33.52)$ \\
Pathological specimens (surgery/biopsy) & $206 / 44$ \\
High-risk factors (cirrhosis/hepatitis B) & $141 / 109$ \\
\hline
\end{tabular}

*, P>0.05. SD, standard deviation; $n$, number; CT, computed tomography; MRI, magnetic resonance imaging; CEUS, contrast-enhanced ultrasound.

\section{Nodule classification and agreement analysis}

CEUS LI-RADS v2017 classified the 259 nodules into 2 LR-1, 4 LR-2, 5 LR-3, 16 LR-4, and 153 LR-5 nodules (Figure 2); 58 LR-M nodules (Figures 3 and 4); and 21 LRTIV nodules, whereas CT/MRI LI-RADS v2018 classified them into 3 LR-1, 4 LR-2, 8 LR-3, 30 LR-4, and 140 LR-5 nodules (Figures 2 and 4); 43 LR-M nodules (Figure 3); and 31 LR-TIV nodules. The Kappa test revealed $\mathrm{K}$ values of 0.659 and 0.630 for CT/MRI and CEUS, respectively
$(\mathrm{P}<0.001)$, indicating that the observers had substantial agreement and moderate agreement between modalities $(\mathrm{K}=0.426, \mathrm{P}<0.001)$.

\section{Diagnostic accuracy for HCC and non-HCC malignancy, and ROC curve analysis}

According to the pathological results (Table 2), the 259 nodules included 233 malignant nodules (172 HCC and 61 non-HCC malignancies) and 26 benign nodules.

In the CEUS LI-RADS v2017 classification, all the 2 LR-1 and 4 LR-2 nodules were benign; the 5 LR-3 nodules included 3 malignant [1 HCC $(1 / 5,20 \%)$ and 2 nonHCC malignancies $(2 / 5,40 \%)]$ and 2 benign nodules; the 16 LR-4 nodules included 13 malignant 8 HCC $(8 / 16$, $50 \%)$ and 5 non-HCC malignancies $(5 / 16,31.3 \%)]$ and 3 benign nodules; the 153 LR-5 nodules included 142 malignant [127 HCC (127/153, 83\%) and 15 non-HCC malignancies $(15 / 153,9.8 \%)]$ and 11 benign nodules; the 58 LR-M nodules included 54 malignant 20 HCC (20/58, $34.5 \%)$ and 34 non-HCC malignancies (34/58, 58.6\%)] and 4 benign nodules; and the 21 LR-TIV included 16 HCC $(16 / 21,76.2 \%)$ and 5 non-HCC malignancies (5/21, $23.8 \%)$.

In the CT/MRI LI-RADS v2018 classification, all the 

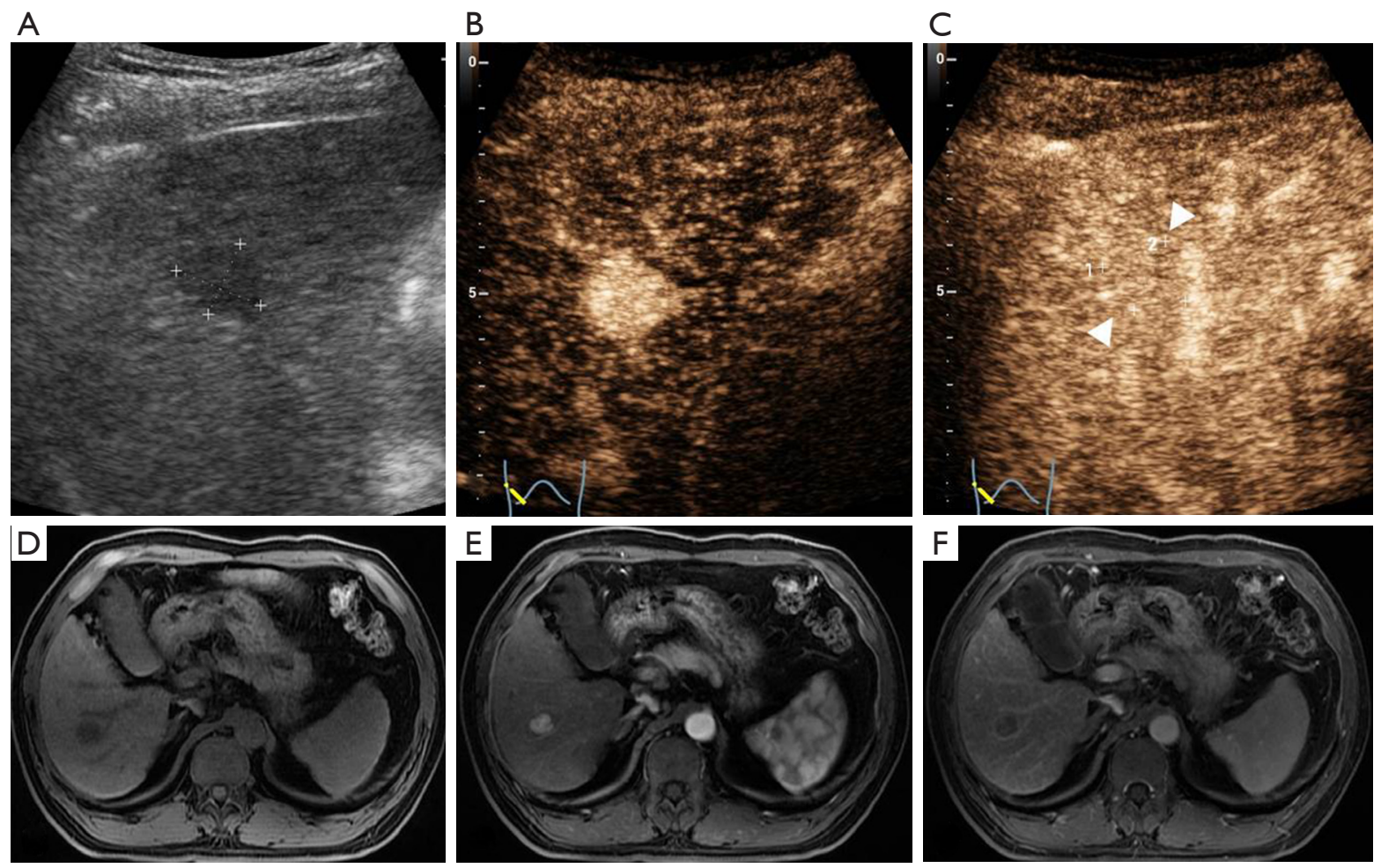

Figure 2 A 55-year-old man with HCC. CEUS showed a hypoechoic nodule on segment 6 of liver with a size of $1.97 \mathrm{~cm}$ (A), obvious hyperenhancement in arterial phase (B) and mild wash out in portal venous phase (C, arrowheads) after the contrast media injection $26 \mathrm{~s}$ and $1 \mathrm{~min} 6 \mathrm{~s}$, respectively. MRI showed a hypointensity nodule on segment 6 of liver with a size of $2.0 \mathrm{~cm}$ (D), hyperenhancement in arterial phase (E) and marked wash out on portal venous phase (F). The nodule was classified as LR-5 both on CEUS and CT/MRI. HCC, hepatocellular carcinoma; CEUS, contrast enhanced ultrasound; CT, computed tomography; MRI, magnetic resonance imaging.

3 LR-1 nodules were benign; the 4 LR-2 nodules included 3 benign and 1 malignant (HCC) nodule; the 8 LR-3 nodules included 3 malignant [2 HCC $(2 / 8,25 \%)$ and 1 non-HCC malignancy $(1 / 8,12.5 \%)]$ and 5 benign nodules; the 30 LR-4 nodules included 24 malignant [18 HCC $(18 / 30,60 \%)$ and 6 non-HCC malignancies $(6 / 30,20 \%)]$ and 6 benign nodules; the 140 LR-5 nodules included 134 malignant [127 HCC (127/140, 90.7\%) and 7 nonHCC malignancies (7/140, 5\%)] and 6 benign nodules; the 43 LR-M nodules included 40 malignant [4 HCC (4/43, $9.3 \%)$ and 36 non-HCC malignancies (36/43, 83.7\%)] and 3 benign nodules; and the $31 \mathrm{LR}-\mathrm{TIV}$ nodules included 20 HCC $(20 / 31,64.6 \%)$ and 11 non-HCC malignancies $(11 / 31,35.4 \%)$.

The ratio of HCC in CT/MRI LR-4 and LR-5 nodules and the ratio of non-HCC malignancy in LR-M nodules were higher than those in CEUS (Table 3). The specificity and PPV of HCC and non-HCC malignancies in LR-5 and LR-M were $85.06 \%$ [95\% confidence interval (CI): $80.72 \%, 89.4 \%$ ], $90.71 \%$ (95\% CI: $87.18 \%, 94.25 \%$ ), 96.46\% (95\% CI: 94.22\%, 98.71\%), $83.72 \%$ (95\% CI: $79.22 \%, 88.22 \%)$ in CT/MRI, and $70.11 \%(95 \% \mathrm{CI}$ : 64.54\%, 75.69\%), $83.01 \%$ (95\% CI: $78.43 \%$, 87.58\%), $87.88 \%$ (95\% CI: $83.9 \%, 91.85 \%$ ), $58.62 \%$ (95\% CI: $52.62 \%, 64.62 \%)$ in CEUS, respectively. The specificity and PPV of CT/MRI LR-5 and LR-M are statistically higher than CEUS $(\mathrm{P}<0.01)$. There were no statistical differences among all NPV (Table 4). Finally, ROC curve analysis (Figure 5) revealed that the areas under curve (AUC) of the latest version of CT/MRI and CEUS LIRADS for diagnosing malignant liver lesions. The AUC of CT/MRI LR-5 and LR-M were 0.794 and 0.777 , and the AUC of CEUS LR-5 and LR-M were 0.720 and 0.718 , respectively. The AUC of CT/MRI LR-5 for HCC is statistically higher than CEUS $(\mathrm{P}<0.05)$. The AUC of CT/MRI LR-M for non-HCC malignancy is higher than 

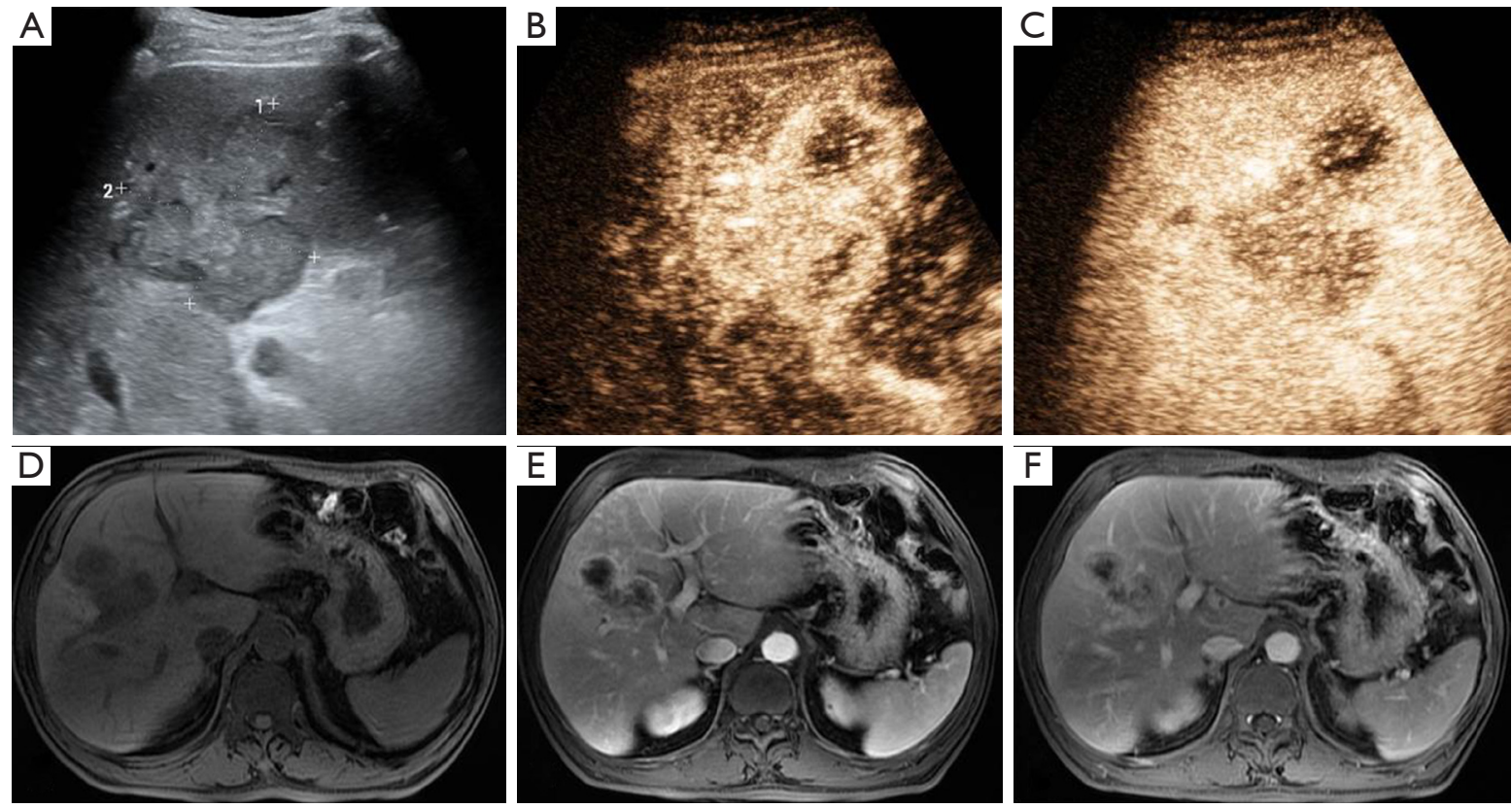

Figure 3 A 60-year-old man with ICC. CEUS showed a hypoechoic mass on segment 5/8 of liver with a size of $5.9 \mathrm{~cm}$ (A), rim- and hyperenhancement in arterial phase (B) and early marked wash out (C) after the contrast media injection 16 and 40 s, respectively. MRI showed a hypointensity mass on segment $5 / 8$ of liver with a size of $4.9 \mathrm{~cm}(\mathrm{D})$, rim-enhancement on arterial phase (E) and continuously enhancement on delay phase (F). The mass was classified as LR-M both on CEUS and CT/MRI. ICC, intrahepatic cholangiocellular carcinoma; CEUS, contrast enhanced ultrasound; CT, computed tomography; MRI, magnetic resonance imaging.

CEUS without statistic difference $(\mathrm{P}>0.05)$.

\section{Discussion}

To improve communication and understanding between radiologists or clinicians, since the release of the first version of LI-RADS in 2011, the ACR has updated LIRADS several times based on user feedback, experience accumulation, emerging evidence, and collaboration with clinical organizations, and released the latest version, including CT/MRI LI-RADS v2018 and CEUS LIRADS v2017 for diagnosis. Application of the latest version of CT/MRI LI-RADS in focal liver lesions in high-risk HCC patients revealed that CT/MRI LI-RADS v2018 has similar specificity but higher sensitivity than v2017 (8). Kim et al. (9) reported that CT/MRI v2018 LR-5 and LR-M can better distinguish HCC from other liver malignancies in patients with cirrhosis. Previous studies $(10,11)$ have shown that CEUS LI-RADS v2017 is an effective and practical diagnostic tool for hepatic nodules, especially the nodules classified as LR-5 and LR-M. Li et al. (12) also demonstrated that CEUS LI-RADS v2017 is a better standardized classification system for high-risk HCC patients, and has good inter-observer agreement. Further, we compared the ACR CT/MRI LI-RADS v2018 with CEUS LI-RADS v2017, and similarly, we found that the two modalities have high diagnostic performance in malignant liver lesions. Meanwhile, the two modalities have substantial agreements inter-observers, and there are moderate agreement inter-modalities. It further illustrates that the two modalities have high application value.

Recently, a study compared CT/MRI LI-RADS v2017 with CEUS LI-RADS v2017; Ding et al. (13) found that the agreement of the two classification modalities is fair. The PPV for HCC in the LR-5 category was comparable between the two. CT/MRI LR-M is superior to CEUS LR-M for the diagnosis of non-HCC malignancies. In this study, we found that the two classification modalities have moderate agreement, which is higher than that reported by Ding et al. (13). We believe that this is related to the simplification and improvement of CT/MRI v2018. However, the specificity of this study is lower than Ding et al. (13), which may be related to the difference in the inclusion and exclusion criteria of patients, and the 

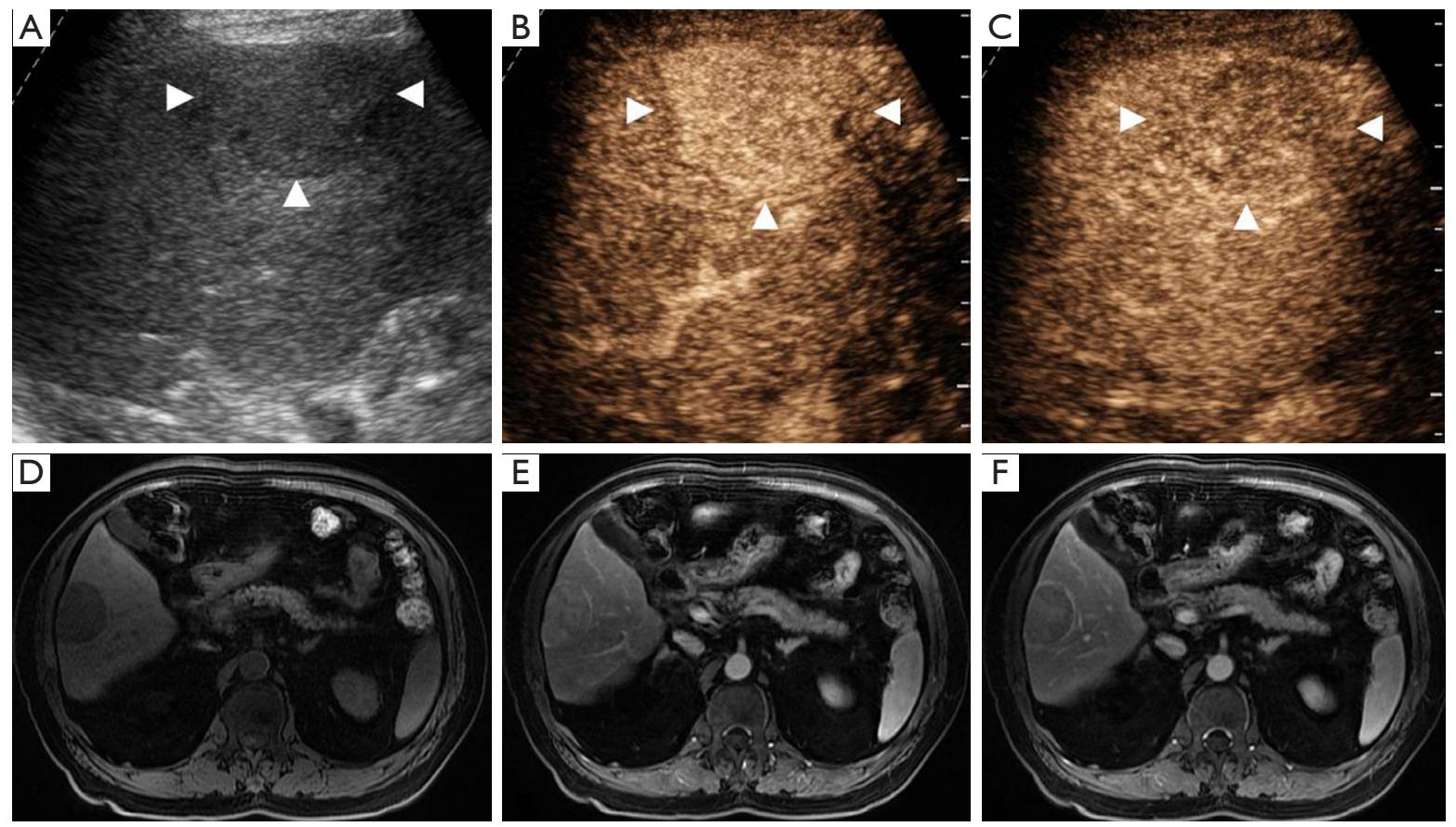

Figure 4 A 82-year-old man with HCC. CEUS showed a hypoechoic mass on segment 5-6 of liver with a size of $4.24 \mathrm{~cm}$ (A, arrowheads), hyperenhancement (B, arrowheads) and early marked wash out (C, arrowheads) in arterial phase after the contrast media injection 28 and 37 s, respectively. MRI showed a hypointensity mass on segment 5-6 of liver with a size of $5.4 \mathrm{~cm}$ (D), hyperenhancement in arterial phase (E) and wash out on portal venous phase (F). The mass was classified as CT/MRI LR-5 and CEUS LR-M. HCC, hepatocellular carcinoma; CEUS, contrast enhanced ultrasound; CT, computed tomography; MRI, magnetic resonance imaging.

contrast media used in contrast enhanced MRI is different. In addition, we found that CT/MRI identified a higher ratio of HCC in LR-4 and LR-5 nodules and non-HCC malignancies in LR-M nodules than did CEUS. When diagnosing HCC and non-HCC malignancies in LR-5 and LR-M nodules, respectively, the specificity, PPV and AUC of CT/MRI were higher than those of CEUS. Although CT/MRI have higher accuracy than CEUS in LR-M nodules, two modalities had a high diagnostic performance, this supports that for some cases, such as (I) lesions that cannot be clearly defined by CT and MRI, (II) patients who are claustrophobic to CT/MRI, (III) and patients allergic or contraindicated (patients with renal failure) to iodine or gadolinium, CEUS can be used as a diagnostic tool undoubtedly. Although American Association for the Study of Liver Diseases (AASLD) now endorses the use of CEUS based on their most recent guidelines, the AASLD and the European Association for the Study of the Liver ever removed CEUS from the guidelines because CEUS may misdiagnose ICC as HCC (14). In recent years, some studies (15-17) have reported that ICC washout occurs earlier, usually within 1 minute (classified as LR-M), and that the hypo-enhancement in the venous phase is more obvious in ICC than in HCC. In this group of LR-M nodules, some nodules started to wash out within 1 minute, but were pathologically proved to be HCC. We believe that this is one of the reasons why the performance of CEUS LR-M is worse than that of CT/MRI in the diagnosis of non-HCC malignancies. A study found that adjusting the wash out time to $45 \mathrm{~s}$ can improve the diagnostic accuracy for LR-M, including that for ICC (18). This indicates that the current criteria for CEUS LR-M may need further discussion and adjustment.

Among the 26 benign nodules, 4 dysplastic nodules, 4 focal nodular hyperplasias (FNH), and 2 angiomyolipomas (AML) were classified into LR-4 or 5 by CT/MRI, whereas 4 dysplastic nodules, 2 cirrhotic nodules, and 2 AML were classified into LR-4 or 5 by CEUS, suggesting that dysplastic nodules, FNH, cirrhotic nodules, and AML have an image overlap with HCC. These factors may decrease the diagnostic specificity and accuracy of LR 4 and LR 5 nodules, and thus further understanding of these lesions is 
Table 2 Pathological results of 259 hepatic nodules

\begin{tabular}{|c|c|c|c|c|c|c|c|c|}
\hline Pathology & \multicolumn{7}{|c|}{ CT/MRI LI-RADS (CEUS LI-RADS) } & Total \\
\hline $\mathrm{HCC}$ & $0[0]$ & $1[0]$ & $2[1]$ & $18[8]$ & $127[127]$ & 4 [20] & $20[16]$ & 172 \\
\hline ICC & $0[0]$ & $0[0]$ & $1[2]$ & $5[4]$ & $6[11]$ & 34 [32] & 8 [5] & 54 \\
\hline cHCC-CC & $0[0]$ & $0[0]$ & $0[0]$ & $1[1]$ & $1[4]$ & $2[1]$ & $2[0]$ & 6 \\
\hline Hemangioma & $3[2]$ & $0[0]$ & $0[0]$ & $0[0]$ & $0[1]$ & $0[0]$ & $0[0]$ & 3 \\
\hline Dysplastic nodules & $0[0]$ & $1[1]$ & $1[0]$ & $2[1]$ & $2[3]$ & $0[1]$ & $0[0]$ & 6 \\
\hline $\mathrm{FNH}$ & $0[0]$ & $2[3]$ & $0[1]$ & $1[0]$ & $3[3]$ & $1[0]$ & $0[0]$ & 7 \\
\hline Chronic inflammation & $0[0]$ & $0[0]$ & $1[1]$ & $0[0]$ & $0[0]$ & $1[1]$ & $0[0]$ & 2 \\
\hline Adenoma & $0[0]$ & $0[0]$ & $1[0]$ & $0[0]$ & $0[1]$ & $0[0]$ & $0[0]$ & 1 \\
\hline Eosinophilic granuloma & $0[0]$ & $0[0]$ & $0[0]$ & $1[0]$ & $0[0]$ & $0[1]$ & $0[0]$ & 1 \\
\hline Liver infarction & $0[0]$ & $0[0]$ & $0[0]$ & $0[0]$ & $0[1]$ & $1[0]$ & $0[0]$ & 1 \\
\hline Total & $3[2]$ & $4[4]$ & $8[5]$ & $30[16]$ & $140[153]$ & $43[58]$ & 31 [21] & 259 \\
\hline
\end{tabular}

Data in square brackets are classifications of CEUS LI-RADS. HCC, hepatocellular carcinoma; ICC, intrahepatic cholangiocellular carcinoma; cHCC-CC, combined hepatocellular cholangiocarcinoma; SHC, sarcomatoid hepatocellular carcinoma; FNH, focal nodular hyperplasia; LIRADS, Liver Imaging Reporting and Data System; TIV, tumor in vein; CT, computed tomography; MRI, magnetic resonance imaging.

Table 3 Malignant ratio of hepatocellular carcinoma (HCC) and non-HCC malignancy between CT/MRI and CEUS Liver Imaging Reporting and Data System (LI-RADS)

\begin{tabular}{lcccc}
\hline \multirow{2}{*}{ Categories } & \multicolumn{2}{c}{ CT/MRI (\%) } & \multicolumn{2}{c}{ CEUS (\%) } \\
\cline { 2 - 4 } \cline { 4 - 5 } LR-4 & HCC & Non-HCC malignancy & HCC & Non-HCC malignancy \\
LR-5 & 60 & 20 & 50 & 31.3 \\
LR-M & 90.7 & 5 & 83 & 9.8 \\
LR-TIV & 9.3 & 83.7 & 34.5 & 58.6 \\
\hline
\end{tabular}

TIV, tumor in vein; CT, computed tomography; MRI, magnetic resonance imaging; CEUS, contrast-enhanced ultrasound.

needed. Meanwhile, there are some limitations in this study. First, some nodules may be downgraded or upgraded after CT/MRI follow-up, only pathologically proven nodules were included, and excluded patients with more than 3 hepatic nodules may cause case selection bias. Second, this study is a single-center retrospective study, and the acquisition of US images was operator-dependent. Third, in this study, only two radiologists classified the nodules, and more observers at different levels are needed to evaluate the classification system more accurately. Lastly, although all patients were primary hepatic nodules, the existence of occult or asymptomatic tumorous lesions in other parts cannot be ruled out.

\section{Conclusions}

ACR CT/MRI LI-RADS v2018 and CEUS LI-RADS v2017 have high application value in primary hepatic nodules in patients with high-risk HCC. Two classification modalities have high diagnostic performance for LR-5 and 
Table 4 Diagnostic accuracy of LR-5 (HCC) and LR-M (non-HCC malignancy) between CT/MRI and CEUS LI-RADS

\begin{tabular}{|c|c|c|c|c|c|c|}
\hline Variables & \multicolumn{3}{|c|}{$\mathrm{HCC}$} & \multicolumn{3}{|c|}{ Non-HCC malignancy } \\
\hline TP & 127 & 127 & NA & 36 & 34 & NA \\
\hline TN & 74 & 61 & NA & 191 & 174 & NA \\
\hline FP & 13 & 26 & NA & 7 & 24 & NA \\
\hline Sensitivity & $0.7384(0.6848,0.7919)$ & $0.7384(0.6848,0.7919)$ & 1.00000 & $0.5902(0.5303,0.6501)$ & $0.5574(0.4969,0.6179)$ & 0.57994 \\
\hline Specificity & $0.8506(0.8072,0.894)$ & $0.7011(0.6454,0.7569)$ & 0.00001 & $0.9646(0.9422,0.9871)$ & $0.8788(0.839,0.9185)$ & 0.00000 \\
\hline PPV & $0.9071(0.8718,0.9425)$ & $0.8301(0.7843,0.8758)$ & 0.00026 & $0.8372(0.7922,0.8822)$ & $0.5862(0.5262,0.6462)$ & 0.00000 \\
\hline NPV & $0.6218(0.5628,0.6809)$ & $0.5755(0.5153,0.6357)$ & 0.41384 & $0.8843(0.8453,0.9232)$ & $0.8657(0.8241,0.9072)$ & 0.36843 \\
\hline
\end{tabular}

HCC, hepatocellular carcinoma; TP, true positive; TN, true negative; FP, false positive; FN, false negative; PPV, positive predictive value; NPV, negative predictive value; NA, not applicable; CT, computed tomography; MRI, magnetic resonance imaging; CEUS, contrastenhanced ultrasound; LI-RADS, Liver Imaging Reporting and Data System.
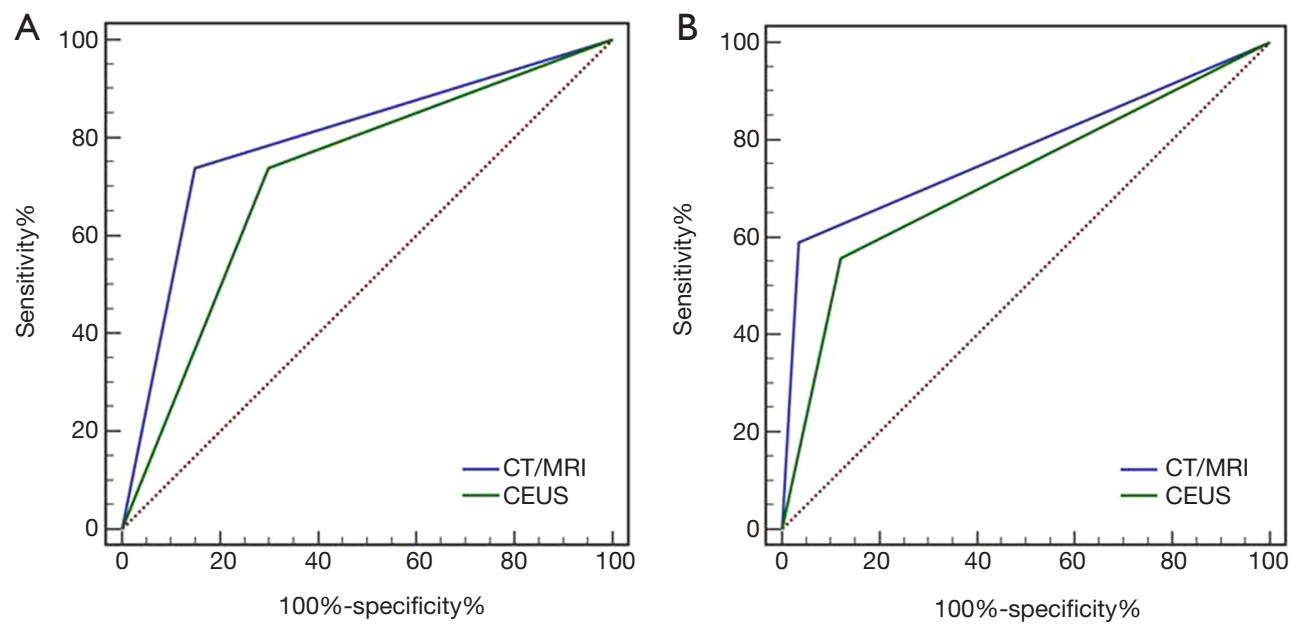

Figure 5 The AUC of two modalities in the diagnosis of liver malignant lesions. The AUC of CT/MRI and CEUS LR-5 (A) in the diagnosis of HCC were 0.794 and 0.720 respectively. The AUC of CT/MRI and CEUS LR-M (B) in the diagnosis of non-HCC malignancy were 0.777 and 0.718 respectively. AUC, areas under the curve; HCC, hepatocellular carcinoma; CEUS, contrast enhanced ultrasound; CT, computed tomography; MRI, magnetic resonance imaging.

LR-M nodules, have substantial agreement inter-observers and moderate agreement inter-modalities, which supports that LI-RADS helps to improve communication and understanding among radiologists or clinicians.

\section{Acknowledgments}

We thank Gold Editing Team for technical support.

Funding: This work was supported by the Clinical Medicine Research Pilot Project of Shanghai Medical College of
Fudan University (grant numbers DGF501022/015), Shanghai Municipal Commission of Science and Technology (grant numbers 19411951200) and Clinical Research Plan of SHDC (grant numbers SHDC2020CR3020A).

\section{Footnote}

Reporting Checklist: The authors have completed the STARD reporting checklist. Available at http://dx.doi.org/10.21037/ atm-21-1035 


\section{Page 10 of 11}

Data Sharing Statement: Available at http://dx.doi. org/10.21037/atm-21-1035

Conflicts of Interest: All authors have completed the ICMJE uniform disclosure form (available at http://dx.doi. org/10.21037/atm-21-1035). The authors have no conflicts of interest to declare.

Ethical Statement: The authors are accountable for all aspects of the work in ensuring that questions related to the accuracy or integrity of any part of the work are appropriately investigated and resolved. The study was conducted in accordance with the Declaration of Helsinki (as revised in 2013). The study was approved by ethics committee of Huashan hospital, Fudan University (No.: KY2021-066) and individual consent for this retrospective analysis was waived.

Open Access Statement: This is an Open Access article distributed in accordance with the Creative Commons Attribution-NonCommercial-NoDerivs 4.0 International License (CC BY-NC-ND 4.0), which permits the noncommercial replication and distribution of the article with the strict proviso that no changes or edits are made and the original work is properly cited (including links to both the formal publication through the relevant DOI and the license). See: https://creativecommons.org/licenses/by-nc-nd/4.0/.

\section{References}

1. Omata M, Cheng AL, Kokudo N, et al. Asia-Pacific clinical practice guidelines on the management of hepatocellular carcinoma: a 2017 update. Hepatol Int 2017;11:317-70.

2. Marrero JA, Kulik LM, Sirlin CB, et al. Diagnosis, Staging, and Management of Hepatocellular Carcinoma: 2018 Practice Guidance by the American Association for the Study of Liver Diseases. Hepatology 2018;68:723-50.

3. European Association for the Study of the Liver. EASL Clinical Practice Guidelines: Management of hepatocellular carcinoma. J Hepatol 2018;69:182-236.

4. Petrick JL, Braunlin M, Laversanne M, et al. International trends in liver cancer incidence, overall and by histologic subtype, 1978-2007. Int J Cancer 2016;139:1534-45.

5. Petrick JL, Kelly SP, Altekruse SF, et al. Future of Hepatocellular Carcinoma Incidence in the United States Forecast Through 2030. J Clin Oncol 2016;34:1787-94.
6. Heimbach JK, Kulik LM, Finn RS, et al. AASLD guidelines for the treatment of hepatocellular carcinoma. Hepatology 2018;67:358-80.

7. McHugh ML. Interrater reliability: the kappa statistic. Biochem Med (Zagreb) 2012;22:276-82.

8. Lee SM, Lee JM, Ahn SJ, et al. LI-RADS Version 2017 versus Version 2018: Diagnosis of Hepatocellular Carcinoma on Gadoxetate Disodium-enhanced MRI. Radiology 2019;292:655-63.

9. Kim YY, Kim MJ, Kim EH, et al. Hepatocellular Carcinoma versus Other Hepatic Malignancy in Cirrhosis: Performance of LI-RADS Version 2018. Radiology 2019;291:72-80.

10. Terzi E, Iavarone $M$, Pompili $M$, et al. Contrast ultrasound LI-RADS LR-5 identifies hepatocellular carcinoma in cirrhosis in a multicenter restropective study of 1,006 nodules. J Hepatol 2018;68:485-92.

11. Zheng W, Li Q, Zou XB, et al. Evaluation of Contrastenhanced US LI-RADS version 2017: Application on 2020 Liver Nodules in Patients with Hepatitis B Infection. Radiology 2020;294:299-307.

12. Li J, Ling W, Chen S, et al. The interreader agreement and validation of contrast-enhanced ultrasound liver imaging reporting and data system. Eur J Radiol 2019;120:108685.

13. Ding J, Long L, Zhang X, et al. Contrast-enhanced ultrasound LI-RADS 2017: comparison with CT/MRI LIRADS. Eur Radiol 2021;31:847-54.

14. Vilana R, Forner A, Bianchi L, et al. Intrahepatic peripheral cholangiocarcinoma in cirrhosis patients may display a vascular pattern similar to hepatocellular carcinoma on contrast-enhanced ultrasound. Hepatology 2010;51:2020-9.

15. Yuan MX, Li R, Zhang XH, et al. Factors Affecting the Enhancement Patterns of Intrahepatic Cholangiocarcinoma (ICC) on Contrast-Enhanced Ultrasound (CEUS) and their Pathological Correlations in Patients with a Single Lesion. Ultraschall Med 2016;37:609-18.

16. Wildner D, Bernatik T, Greis C, et al. CEUS in hepatocellular carcinoma and intrahepatic cholangiocellular carcinoma in 320 patients - early or late washout matters: a subanalysis of the DEGUM multicenter trial. Ultraschall Med 2015;36:132-9.

17. Liu GJ, Wang W, Lu MD, et al. Contrast-Enhanced Ultrasound for the Characterization of Hepatocellular Carcinoma and Intrahepatic Cholangiocarcinoma. Liver 
Cancer 2015;4:241-52.

18. Li F, Li Q, Liu Y, et al. Distinguishing intrahepatic cholangiocarcinoma from hepatocellular carcinoma in patients with and without risks: the evaluation of the

Cite this article as: Lv K, Cao X, Dong Y, Geng D, Zhang J. CT/MRI LI-RADS version 2018 versus CEUS LIRADS version 2017 in the diagnosis of primary hepatic nodules in patients with high-risk hepatocellular carcinoma. Ann Transl Med 2021;9(13):1076. doi: 10.21037/atm-21-1035
LR-M criteria of contrast-enhanced ultrasound liver imaging reporting and data system version 2017. Eur Radiol 2020;30:461-70. 